\title{
Adherence to riluzole in patients with amyotrophic lateral sclerosis: an observational study
}

This article was published in the following Dove Press journal:

Neuropsychiatric Disease and Treatment

\section{Alessandro Introna \\ Eustachio D'Errico \\ Boris Modugno \\ Antonio Scarafino \\ Angela Fraddosio \\ Eugenio Distaso \\ Irene Tempesta \\ Antonella Mastronardi \\ Isabella Laura Simone \\ Neurology Unit, Department of Basic Medical Sciences, Neurosciences and Sense Organs, University of Bari \\ “Aldo Moro," Bari, Italy}

Correspondence: Isabella Laura Simone Department of Basic Medical Sciences, Neurosciences and Sense Organs, University of Bari “Aldo Moro," Piazza Giulio Cesare II, 70I00 Bari, Italy Tel +39805478519

Email isabellalaura.simone@uniba.it
Objective: Riluzole is the first drug approved to treat amyotrophic lateral sclerosis (ALS) Recently, an oral suspension (OS) of riluzole was made available. Thus, the aim of our study was to evaluate the adherence to 2 formulations of riluzole in patients with ALS.

Patients and methods: We enrolled 45 consecutive patients with ALS. At disease diagnosis, riluzole was prescribed in 2 different formulations depending on the severity of dysphagia (27/45 patients received tablets and 18/45 patients received OS). Side effects (SEs) and treatment adherence were investigated using a clinical questionnaire including the CMorisky 8-item Medication Adherence Questionnaire.

Results: Gastroenteric complaints were the most frequent SEs (58\% in the tablet group and $48 \%$ in the OS group), followed by those at the nervous system ( $29 \%$ and $40 \%$, respectively). No serious SEs related to treatment were reported. The rate of adherence to riluzole was independent of the formulation of the drug and consistent with other medications assumed for comorbidities $(p=0.004)$. In the tablet group, low adherence was caused by SEs in $55.6 \%$ and by dysphagia in $44.4 \%$ of patients. In the OS group, SEs caused low adherence in $75 \%$ of patients. Independently of the drug formulation, patients with high or medium adherence to riluzole had a higher progression rate ( $p=0.002$ and $p=0.009$, respectively) and a shorter time to generalization (TTG; $p=0.01$ ), compared to those with low adherence.

Conclusion: Gastroenteric symptoms were the most frequent SE related to tablet as well as OS. The rate of adherence was independent of the formulation of riluzole and the number of medications assumed for comorbidities, and it was consistent with the severity of the disease. The low adherence was caused by dysphagia and SEs in the tablet group, whereas it was caused prevalently by SEs in the OS group.

Keywords: adherence, riluzole, oral suspension, tablet, side effects

\section{Introduction}

Amyotrophic lateral sclerosis (ALS) is a devastating progressive neurodegenerative disease, which causes death within 3-4 years from symptom onset. ${ }^{1}$ The etiology of disease is still not well understood, resulting in a lack of really effective diseasemodifying therapies. To date, riluzole is the only drug approved to treat ALS; riluzole has been demonstrated to delay invasive mechanical ventilation or death for several months. ${ }^{2-5}$ Only recently, a new drug, edaravone, was approved by the Food and Drug Administration (FDA). ${ }^{6}$ Riluzole blocks glutamate-mediated excitatory neurotransmission attenuating excitotoxicity, one of the proposed pathophysiological pathways of neuronal death. Riluzole tablet was the only available formulation until 2014, when the oral suspension (OS) formulation has been approved. Considering that dysphagia 
is a symptom experienced by the majority of patients during the natural history of disease, one of the reasons for therapy discontinuation is just the tablet formulation therapy. Therefore, the OS riluzole $5 \mathrm{mg} / \mathrm{mL}$ certainly provides more advantages to the dysphagic patients, thus improving therapeutic outcome and quality of life. ${ }^{7}$

Previous studies explored the safety and tolerability of riluzole therapy in patients who have been included in one of the two international, multicenter, randomized, double-blind, placebo-controlled trials ${ }^{8}$ or who have not been eligible for clinical trials but had access to drug, while it was under the review of regulatory agencies. ${ }^{9}$ The overall tolerability of riluzole was good, and the treatment was indicated for all patients with ALS, except for those with hepatic disease and/or who had an increase in transaminase ( $>3$, the upper limit). ${ }^{10}$

An important worldwide public health problem in the management of chronic diseases is the medication adherence and persistence. Adherence is defined as "the extent to which a patient acts in accordance with the prescribed interval and dose of a dosing regimen." "11 Adherence has been studied in many chronic illnesses, such as HIV infection, ${ }^{12}$ hypertension, ${ }^{13}$ diabetes, ${ }^{14}$ epilepsy, ${ }^{15}$ multiple sclerosis ${ }^{16}$ and psychiatric disorders. ${ }^{17}$ Overall, an average adherence of only $50 \%$ is indicated by the World Health Organization among patients with chronic diseases. ${ }^{18}$ The adherence to riluzole therapy in patients with ALS has not been evaluated yet.

The aim of our observational study was to examine the adherence to riluzole in the 2 available formulations in patients with ALS and the potential factors that can influence it.

\section{Patients and methods}

\section{Study population}

We enrolled 45 consecutive patients with ALS referring to the Tertiary Centre of Motor Neuron Disease of our department from August 2016 to January 2017, followed until February 2017. The date of diagnosis ranged from January 2011 to August 2016.

All the included patients were prescribed with riluzole and vitamin $\mathrm{E}$ at disease diagnosis. Until 2014, all the patients received riluzole tablet; after 2014, either tablet or OS of riluzole was prescribed according to the presence and severity of dysphagia. At the time of observation, 21 patients had definite, 7 probable, 4 laboratory-supported probable and 13 possible ALS, according to El Escorial-r criteria. ${ }^{19}$ After obtaining informed consent, a clinical questionnaire, specifically structured for the study in object, was administered by a neurologist to all patients in the presence of a family member or caregiver. Demographic and clinical data, including actual age, sex, age at symptom onset, site of onset (bulbar or spinal), disease duration from symptom onset to interview, symptom onset to therapy start interval (OTI), duration of therapy at interview, follow-up duration, ALS Functional Rating Scale - revised (ALSFRS-r) and Manual Muscle Testing - medium (MMT-m) at observation, time to generalization (TTG; time interval between disease spreading from spinal to bulbar district or vice versa) and progression rate (48 - ALSFRS-r)/month of disease duration were collected (Supplementary material). Information on the specific treatment for ALS (tablets or OS of riluzole), their side effects (SEs) and other co-medications were recorded (see Figure S1). Patients taking OS of riluzole reported also a qualitative opinion (pleasant, unpleasant or indifferent) about aroma, color, consistency and flavor of the drug. Finally, Italian version of CMorisky 8-item Medication Adherence Questionnaire was used to assess the adherence to riluzole and other concomitant medications. ${ }^{20-23}$

The study was approved by the local independent ethics committee of the Regional University Hospital of Bari, Italy.

\section{Statistical analysis}

Statistical analysis was performed using IBM SPSS Statistics 20 (IBM Corporation, Armonk, NY, USA). Group comparison was computed using Mann-Whitney/Wilcoxon test for 2 independent samples and with $\chi^{2}$ test or Fisher's exact text for nominal variables. The relationship between the rate of adherence to riluzole and the number of medications or clinical parameters was assessed by multinomial logistic regression. Statistical significance was considered when $p$-values were $<0.05$.

\section{Results}

\section{Clinical features}

At the time of diagnosis, $27 / 45$ patients (17 males) received tablets and 18/45 patients received OS (10 males) of riluzole. The bulbar onset was significantly more frequent in the OS group (14/18 in the OS group and 4/27 in the tablet group), and all but 2 patients of this group reported dysphagia (13/18 in the OS group and 3/27 in the tablet group) at diagnosis. In the tablet group, although a slight dysphagia at diagnosis was reported in 11 patients, it did not influence the intake of tablets. The 2 groups did not differ in terms of age at disease onset, OTI, duration of riluzole assumption at the time of interview, time of interview from therapy initiation, duration of disease from symptom onset to interview, follow-up duration, ALSFRS-r and TTG, whereas MMT-m was lower in the tablet group (Table 1).

During the follow-up (range 1.5-6months), 8 patients shifted from tablets to OS and 4 patients shifted from OS to tablets, 
Table I Clinical features of patients who received tablet and OS of riluzole

\begin{tabular}{|c|c|c|c|}
\hline Clinical features & Tablet of riluzole $(n=27)$ & OS of riluzole $(n=18)$ & $p$-value \\
\hline $\operatorname{Sex}(M / F)$ & $17 / 10$ & $10 / 8$ & ns \\
\hline Age at onset & $61.84 \pm 9.97$ & $66.80 \pm 9.39$ & ns \\
\hline Spinal/bulbar at onset & $23 / 4$ & $4 / 14$ & $0.00002 *$ \\
\hline Dysphagia at diagnosis & I | (40.7\%) & 16 (88.9\%) & $0.001 * *$ \\
\hline Disease duration from symptom onset to interview & $49.9 \pm 96.48$ & $29.4 \pm 14.1$ & ns \\
\hline OTI (months) & $13.99 \pm 9.03$ & $19.47 \pm 13.27$ & ns \\
\hline Duration of therapy at interview (months) & $14.57 \pm 22.38$ & $9.1 \pm 7.05$ & ns \\
\hline Follow-up period (months) & $3.7 \pm 0.98$ & $4.3 \pm 1.2$ & ns \\
\hline Time to interview from therapy initiation (months) & $19.57 \pm 26.28$ & $10.87 \pm 7.3$ & ns \\
\hline MMT-m at interview & $7.8 \pm 1.13$ & $8.82 \pm 1.35$ & $0.0009^{\wedge}$ \\
\hline ALSFRS-r at interview & $31.74 \pm 7.57$ & $34.17 \pm 7.27$ & ns \\
\hline TTG (months) & $14.36 \pm 13.78$ & $10.79 \pm 10.53$ & ns \\
\hline Progression rate at interview & $0.7 I \pm 0.56$ & $0.59 \pm 0.60$ & ns \\
\hline
\end{tabular}

Notes: $* \chi^{2}=17.8 ; * * \chi^{2}=10.4 ;{ }^{\wedge}$ Kruskal-Wallis $\mathrm{H}=10.9$.

Abbreviations: ALSFRS-r, ALS Functional Rating Scale - revised; MMT-m, Manual Muscle Testing - medium; ns, not significant; OS, oral suspension; OTI, symptom onset to therapy start interval; TTG, time to generalization.

as given in the "Change of riluzole formulation" section. The 2 groups did not differ in terms of OTI, duration of riluzole assumption, TTG, age at onset, ALSFRS-r, MMT-m and progression rate at interview.

\section{Opinion about OS of riluzole}

A total of 26 patients on OS of riluzole (18 patients since the study inclusion and 8 patients who shifted from tablet to OS during the follow-up) reported a qualitative opinion about the OS. Fragrance, color and consistency were considered not relevant in the majority of patients. The flavor was unpleasant in 14 patients $(53.8 \%), 12$ of whom considered the flavor was very strongly unpleasant (Figure 1).

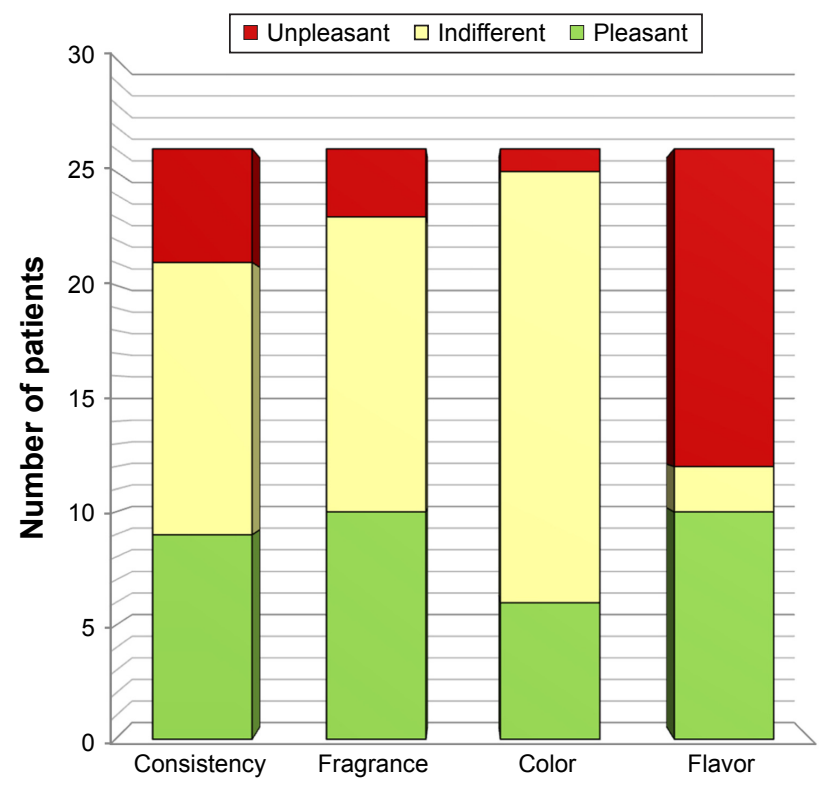

Figure I Qualitative opinion about organoleptic characteristics of OS of riluzole. Abbreviation: OS, oral suspension.

\section{SE profile}

In patients in both the tablet and OS groups, the most frequent SE involved the gastrointestinal apparatus. No serious SEs related to treatment were reported. Figure 2 shows the frequency of SE for apparatus.

The SEs occurring in $>10 \%$ of patients were dysgeusia $(22.2 \%)$ and abdominal pain $(11.1 \%)$ in the tablet group, whereas oral paresthesia (38.9\%), dysgeusia (33.3\%) and nausea $(11.1 \%)$ in the OS group. A detailed list of SEs recorded by patients is reported in Table 2 .

In patients who changed the formulation therapy during the follow-up period, gastroenteric and neurological symptoms remained the most frequent SEs with both the formulations (data not shown).

\section{Change of riluzole formulation}

During the follow-up, 8 tablet-group patients shifted to OS riluzole, 7 of whom ( $87.5 \%$ ) experienced a severe worsening of dysphagia that limited the assumption of the tablets; only 1 patient experienced a severe gastrointestinal SE.

Four patients shifted from OS to tablet of riluzole for SEs affecting both the gastrointestinal and nervous systems ( $50 \%$, respectively). Two of these patients considered that the flavor of the OS was unpleasant.

Four out of 45 patients $(8.9 \%)$ permanently discontinued the riluzole for SEs affecting both the gastrointestinal and nervous systems, some of them even after changing formulation.

\section{Adherence}

The rate of adherence (high, medium, low) to riluzole therapy was independent of the formulation of the $\operatorname{drug}\left(\chi^{2}, p=\mathrm{ns}\right)$. 


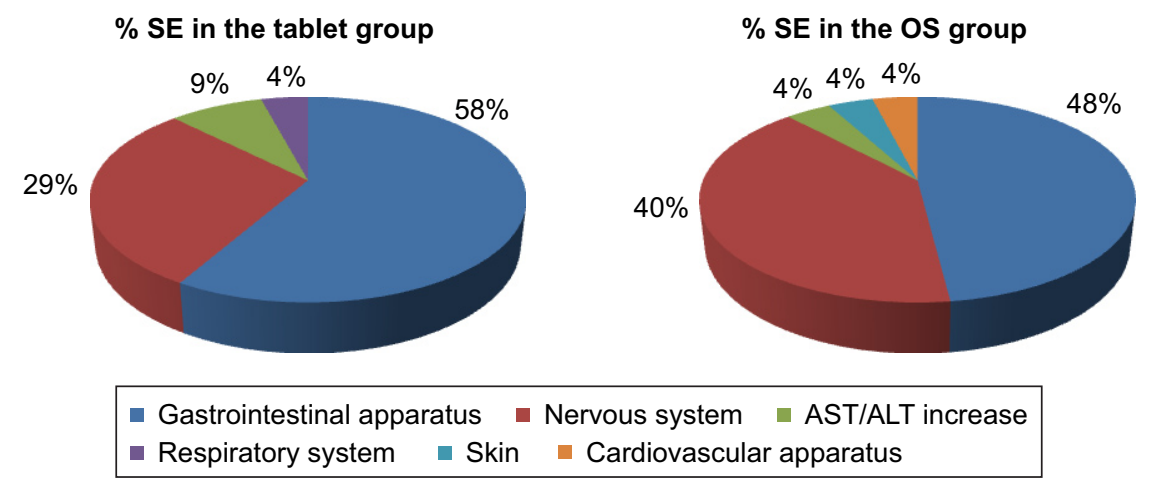

Figure 2 Percentages of SEs for apparatus in the riluzole tablet and OS groups.

Abbreviations: ALT, alanine aminotransferase; AST, aspartate aminotransferase; OS, oral suspension; SE, side effect.

Even considering only the patients with bulbar onset and dysphagia at diagnosis, the rate of adherence to riluzole therapy was independent of the formulation of the drug ( $p=\mathrm{ns}$, Fisher's exact test).

When examining the adherence to the specific formulation of the riluzole, 9 patients showed high, 9 medium and 9 low adherence to riluzole tablet (33.3\%, respectively). The low adherence to riluzole tablets was related to SEs (mostly gastroenteric) in 5 out of 9 patients $(55.6 \%)$ who considered them so intolerable to stop therapy ( 2 patients), to change the formulation (1 patient) or to reduce the posology ( 2 patients), whereas in the remaining 4 patients $(44.4 \%)$, it was related

Table 2 Detailed list of SEs reported by patients (\%)

\begin{tabular}{|c|c|c|}
\hline & $\begin{array}{l}\text { Tablet of riluzole } \\
(n=27)(\%)\end{array}$ & $\begin{array}{l}\text { OS of riluzole } \\
(n=18)(\%)\end{array}$ \\
\hline \multicolumn{3}{|l|}{ Gastrointestinal apparatus } \\
\hline Burning mouth syndrome & 0 & I (5.6) \\
\hline Hypersalivation & I (3.7) & I (5.6) \\
\hline Dyspepsia & I (3.7) & I (5.6) \\
\hline Nausea & $2(7.4)$ & $2(I I . I)$ \\
\hline Diarrhea & I (3.7) & 0 \\
\hline Abdominal pain & $3(11.1)$ & I (5.6) \\
\hline Dysgeusia & $6(22.2)$ & $6(33.3)$ \\
\hline \multicolumn{3}{|l|}{ Nervous system } \\
\hline Headache & I (3.7) & I (5.6) \\
\hline Drowsiness & I (3.7) & I (5.6) \\
\hline Asthenia & $2(7.4)$ & I (5.6) \\
\hline Dizziness & I (3.7) & 0 \\
\hline Limb paresthesia & I (3.7) & 0 \\
\hline Oral paresthesia & 0 & $7(38.9)$ \\
\hline Cramps & I (3.7) & 0 \\
\hline AST/ALT increase ( $\geq 2$ ULN) & $2(7.4)$ & I (5.6) \\
\hline \multicolumn{3}{|l|}{ Respiratory system } \\
\hline Dyspnea & I (3.7) & 0 \\
\hline \multicolumn{3}{|l|}{ Skin } \\
\hline Rash & 0 & I (5.6) \\
\hline \multicolumn{3}{|l|}{ Cardiovascular apparatus } \\
\hline Arrhythmia & 0 & I (5.6) \\
\hline
\end{tabular}

Abbreviations: ALT, alanine aminotransferase; AST, aspartate aminotransferase; OS, oral suspension; SE, side effect; ULN, upper limit of normal. to dysphagia. In medium adherence group, a progressive dysphagia limited the adherence in 3 patients, who shifted to OS of riluzole during the follow-up. In the high adherence group, the SEs, if present, were considered tolerable. In the OS group, 8 patients had low adherence to riluzole (44.4\%) prevalently (75\%) depending on SEs affecting the nervous system or gastrointestinal apparatus, which caused the change of formulation in 3 patients. Seven patients showed medium adherence (38.9\%) and $3(16.7 \%)$ high adherence. Seven out of 18 patients who received OS, independently of the rate of adherence, defined the OS as unpleasant flavor. In the subgroup of patients with bulbar onset and dysphagia, the low adherence was mainly related to SEs independently of the formulation of riluzole.

Considering the therapies assumed for comorbidities, the rate of adherence to riluzole was consistent with other medications $\left(\chi^{2} 15.4, p=0.004\right)$. In addition, no relationship was found between the number of medications and the rate of adherence to riluzole ( $p=\mathrm{ns}$ ).

When the relationships between the rates of adherence to riluzole and clinical features were evaluated, patients with high or medium adherence to riluzole, independently of the formulation, had more likely a higher progression rate (pseudo $\mathrm{R}^{2}=0.179, p=0.002$ and $p=0.009$, respectively) and a shorter TTG (pseudo $\mathrm{R}^{2}=0.391$ and $p=0.01$ for both), in comparison with those with low adherence.

\section{Discussion}

This is the first study that evaluates the adherence to the different formulations of riluzole in patients with ALS.

The adherence to medication could be influenced by different factors classifiable into those related to medication (route of administration and type of formulation, polypharmacy, SE), demographic features (sex, age, social class and ethnic group), disease characteristics (severity and clinical 
parameters) and patient's perception and attitude (treatment of asymptomatic diseases, patients' belief in the benefit of therapy). ${ }^{24}$ Nonadherence to medication is a crucial problem in chronic diseases, ${ }^{25}$ because patients who do not adhere to or persist with therapy are unlikely to receive full benefits of treatment and to achieve better clinical outcomes. ${ }^{16}$

In chronic diseases, findings on the influence of route of administration/drug formulation on the adherence to treatment are conflicting. ${ }^{26,27}$ The first finding of our study is that the rate of adherence or persistence to riluzole was not affected by the formulation of the therapy. The lack of relationship between the rate of adherence and the 2 formulations of riluzole was confirmed also in the subgroup of patients with bulbar onset and dysphagia at the diagnosis of disease, suggesting that the dysphagia was not the main factor limiting the intake of tablet or OS of riluzole.

Although half of the patients that assumed oral suspension of riluzole considered the drug was unpleasant to taste, this was not a reason for low adherence. Instead, the low adherence was mainly due to SEs in both treatment groups, in $75 \%$ of cases with OS and in $55.6 \%$ with tablet formulation. However, in the tablet group, the progression of the disease, in particular the involvement of bulbar functions with severe dysphagia, was responsible for the low adherence in a substantial proportion of patients (44.4\%). Examining the complained SEs, the most frequent disturbances were at the gastrointestinal system in both treatment groups, followed by those at the nervous system in the OS group. Oral paresthesia was reported only in patients taking OS in a high percentage (38.9\%), although the novel composition of the aqueous vehicle was designed to minimize the local anesthetic effect of the drug in the mouth. ${ }^{7}$ Our findings disagree with the technical data sheet of riluzole ${ }^{28}$ that reports oral paresthesia generally in a low percentage of patients $(1.6 \%)$, reflecting the frequency of SE obtained in clinical trials and observational studies. ${ }^{2,3,8-10}$ Furthermore, in our study, the frequencies of liver function abnormalities, asthenia and nausea were relatively low, with respect to other reports. ${ }^{2,3,8}$

In 4 out of 45 patients $(8.9 \%)$, the SEs were responsible for a permanent riluzole interruption. This percentage was lower than the discontinuation rates reported in randomized studies $^{2,3}$ and quite similar to that in observational studies. ${ }^{8-10}$ Although low, this percentage may be remarkable considering that riluzole is one of the few therapies approved for this disease, unlike other diseases as multiple sclerosis in which several drugs of almost equal efficacy are available. ${ }^{29}$

Another finding of our study is that the polypharmacy did not influence the adherence to riluzole. We speculate that the severity of the ALS could induce a higher adherence such as reported for other severe diseases (ie, cancer or HIV/ AIDS). ${ }^{30,31}$

An interesting result is that the adherence to the therapy was directly related to the progression rate of the disease and inversely related to the TTG, suggesting that a rapid diffusion of the disease leads to take therapy with greater continuity, hoping to stop the disease progression. This finding was consistent with the literature data. ${ }^{32,33}$

Although there are some strengths of this study, there are some limitations to be considered: 1) the relative small number of patients and 2) the lack of information about the cognitive profile of patients who could influence the adherence to the therapies. However, we feel that this aspect has not biased our results because the questionnaire was administered in the presence of a family member or caregiver who could confirm the accuracy of the answers provided by the patient.

The evaluation of the adherence to riluzole is relevant; although for a correct interpretation of our results, further studies with a higher number of patients, taking into account their clinical profile, are needed.

\section{Acknowledgments}

We thank Dr Antonio Leo who was involved in the conception and drawing up of the clinical questionnaire. Use of the CMMAS is protected by US copyright laws. Permission for use is required and was obtained for this study. A license agreement is available from Donald E Morisky, MMAS Research LLC, 14725 NE 20th Street, Bellevue, WA 98007, USA, or from dmorisky@gmail.com.

\section{Author contributions}

Isabella Laura Simone and Eustachio D'Errico conceived and designed the study. Eustachio D'Errico, Alessandro Introna, Boris Modugno and Isabella Laura Simone performed the experiments. Alessandro Introna, Eustachio D'Errico, Boris Modugno and Isabella Laura Simone analyzed the data. Eustachio D’Errico, Alessandro Introna, Eugenio Distaso, Antonio Scarafino, Angela Fraddosio, Irene Tempesta and Antonella Mastronardi contributed to acquisition of data, Alessandro Introna and Isabella Laura Simone drafted the paper. Isabella Laura Simone drafted/revised the manuscript for content, including medical writing for content, study concept or design, analysis or interpretation of data, statistical analysis and study supervision and coordination. All authors contributed toward data analysis, drafting and critically revising the paper and agree to be accountable for all aspects of the work. 


\section{Disclosure}

The authors report no conflicts of interest in this work.

\section{References}

1. van Es MA, Hardiman O, Chio A, et al. Amyotrophic lateral sclerosis. Lancet. 2017;390(10107):2084-2098.

2. Bensimon G, Lacomblez L, Meininger V; ALS/Riluzole Study Group. A controlled trial of riluzole in amyotrophic lateral sclerosis. $N$ Engl J Med. 1994;330(9):585-591.

3. Lacomblez L, Bensimon G, Leigh PN, et al. A confirmatory doseranging study of riluzole in ALS. ALS/Riluzole Study Group-II. Neurology. 1996;47(6 Suppl 4):S242-S250.

4. Lacomblez L, Bensimon G, Leigh PN, Guillet P, Meininger V; for the Amyotrophic Lateral Sclerosis/Riluzole Study Group II. Doseranging study of riluzole in amyotrophic lateral sclerosis. Lancet. 1996; 347(9013):1425-1431.

5. Zoccolella S, Beghi E, Palagano G, et al; SLAP Registry. Riluzole and amyotrophic lateral sclerosis survival: a population-based study in southern Italy. Eur J Neurol. 2007;14(3):262-268.

6. Traynor K. FDA approves edaravone for amyotrophic lateral sclerosis. Am J Health Syst Pharm. 2017;74(12):868.

7. Dyer AM, Smith A. Riluzole $5 \mathrm{mg} / \mathrm{mL}$ oral suspension: for optimized drug delivery in amyotrophic lateral sclerosis. Drug Des Devel Ther. 2016;11:59-64.

8. Lacomblez L, Bensimon G, Leigh PN, et al; ALS Study Groups I and II. Long-term safety of Riluzole in amyotrophic lateral sclerosis. Amyotroph Lateral Scler Other Motor Neuron Disord. 2002;3(1):23-29.

9. Debove C, Zeisser P, Salzman PM, Powe LK, Truffinet P. The Rilutek (Riluzole) Global Early Access Programme: an open-label safety evaluation in the treatment of amyotrophic lateral sclerosis. Amyotroph Lateral Scler Other Motor Neuron Disord. 2001;2(3):153-158.

10. Bensimon G, Doble A. The tolerability of riluzole in the treatment of patients with amyotrophic lateral sclerosis. Expert Opin Drug Saf. 2004;3(6):525-534.

11. Cramer JA, Roy A, Burrell A, et al. Medication compliance and persistence: terminology and definitions. Value Health. 2008;11(1):44-47.

12. Cook PF, Schmiege SJ, Starr W, Carrington JM, Bradley-Springer L. Prospective state and trait predictors of daily medication adherence behavior in HIV. Nurs Res. 2017;66(4):275-285.

13. Haley WE, Gilbert ON, Riley RF, et al; SPRINT Study Research Group. The association between self-reported medication adherence scores and systolic blood pressure control: a SPRINT baseline data study. $J$ Am Soc Hypertens. 2016;10(11):857.e-864.e.

14. Raj GD, Hashemi Z, Soria Contreras DC, et al. Adherence to diabetes dietary guidelines assessed using a validated questionnaire predicts glucose control in individuals with type 2 diabetes. Can J Diabetes. Epub 2017 Jun 22.

15. Getnet A, Woldeyohannes SM, Bekana L, et al. Antiepileptic drug nonadherence and its predictors among people with epilepsy. Behav Neurol. 2016;2016:3189108.

16. Patti F. Optimizing the benefit of multiple sclerosis therapy: the importance of treatment adherence. Patient Pref Adherence. 2010;4:1-9.

17. Sendt KV, Tracy DK, Bhattacharyya S. A systematic review of factors influencing adherence to antipsychotic medication in schizophreniaspectrum disorders. Psychiatry Res. 2015;225(1-2):14-30.
18. Sabaté E. Adherence to Long-Term Therapies: Evidence for Action [dissertation]. Geneva, Switzerland: World Health Organization; 2003.

19. Brooks BR, Miller RG, Swash M, Munsat TL; World Federation of Neurology Research Group on Motor Neuron Diseases. El Escorial revisited: revised criteria for the diagnosis of amyotrophic lateral sclerosis. Amyotroph Lateral Scler Other Motor Neuron Disord. 2000;1(5): 293-299.

20. Morisky DE, Ang A, Krousel-Wood M, Ward HJ. Predictive validity of a medication adherence measure in an outpatient setting. $J$ Clin Hypertens (Greenwich). 2008;10(5):348-354.

21. Fabbrini G, Abbruzzese G, Barone P, et al; REASON Study Group. Adherence to anti-Parkinson drug therapy in the "REASON" sample of Italian patients with Parkinson's disease: the linguistic validation of the Italian version of the "Morisky Medical Adherence Scale- 8 items". Neurol Sci. 2013;34(11):2015-2022.

22. Krousel-Wood MA, Islam T, Webber LS, Re RS, Morisky DE, Muntner P. New medication adherence scale versus pharmacy fill rates in seniors with hypertension. Am J Manag Care. 2009;15(1):59-66.

23. Morisky DE, DiMatteo MR. Improving the measurement of selfreported medication non-adherence: final response. J Clin Epidemi. 2011;64:258-263.

24. Brown MT, Bussell JK. Medication adherence: WHO cares? Mayo Clin Proc. 2011;86(4):304-314.

25. Yeaw J, Benner JS, Walt JG, Sian S, Smith DB. Comparing adherence and persistence across 6 chronic medication classes. J Manag Care Pharm. 2009;15(9):728-740.

26. Fautrel B, Balsa A, Van Riel P, et al. Influence of route of administration/ drug formulation and other factors on adherence to treatment in rheumatoid arthritis (pain related) and dyslipidemia (non-pain related). Curr Med Res Opin. 2017;33(7):1231-1246.

27. Hankin CS, Cox L, Lang D, et al. Medical costs and adherence in patients receiving aqueous versus pressurized aerosol formulations of intranasal corticosteroids. Allergy Asthma Proc. 2012;33(3):258-264.

28. U.S. Food and Drug Administration. RILUTEK ${ }^{\circledR}$. Silver Spring: U.S. Food and Drug Administration. Available from: https://www. accessdata.fda.gov/drugsatfda_docs/label/2009/020599s011s012lbl. pdf. Accessed November 20, 2017.

29. Fogarty E, Schmitz S, Tubridy N, Walsh C, Barry M. Comparative efficacy of disease-modifying therapies for patients with relapsing remitting multiple sclerosis: systematic review and network meta-analysis. Mult Scler Relat Disord. 2016;9:23-30.

30. Donovan KA, Greene PG, Shuster JL, Partridge EE, Tucker DC. Treatment preferences in recurrent ovarian cancer. Gynecol Oncol. 2002; 86(2):200-211.

31. Gao X, Nau DP, Rosenbluth SA, Scott V, Woodward C. The relationship of disease severity, health beliefs and medication adherence among HIV patients. AIDS Care. 2000;12(4):387-398.

32. Park H, Rascati KL, Prasla K, McBayne T. Evaluation of health care costs and utilization patterns for patients with gout. Clin Ther. 2012;34(3): 640-652.

33. Sarawate CA, Brewer KK, Yang W, et al. Gout medication treatment patterns and adherence to standards of care from a managed care perspective. Mayo Clin Proc. 2006;81(7):925-934. 


\section{Supplementary materials}

Questionnaire for evaluation of "adherence to riluzole in patients with amyotrophic lateral sclerosis" Date of interview

Neurologist:

Number:

Clinical File number:

\section{Informed consent:}

Yes |__ Nol_ $\mid$

If yes, proceed to fill in the questionnaire.

If no, please indicate, where possible, the reason for the refusal:

\section{Anagraphic and socio-demographic data:}

\begin{tabular}{|l|l|}
\hline Last name & \\
\hline Name & \\
\hline Date of birth & I__ \\
\hline City of residence & \\
\hline Country & \\
\hline Where he/she lives (own home or other) & \\
\hline Year of first visit at Motor Neuron Centre & \\
\hline
\end{tabular}

\section{Title of study:}

$\square$ University degree

$\square$ High school license

$\square$ Middle school license
Primary school license

$\square$ None - able to write/read

$\square$ None - not able to write/read

\section{Caregiver:}

Age Sex |_ $|M|$ | F Does he/she live with patient 24 hours/day? I_ Yes |_ No

\section{Caregiver's relation to patient:}

1. Partner

2. Wife/husband

3. Father/mother

4. Brother/sister

5. Nephew

6. Friend/neighbor

7. Other

Figure SI (Continued) 


\section{Amyotrophic lateral sclerosis clinical features:}

\begin{tabular}{|l|l|}
\hline El-Escorial-r category at interview & \\
\hline Disease symptoms onset (date/symptoms) & \\
\hline Diffusion (date/symptoms) & \\
\hline Generalization (date/symptoms) & \\
\hline Date of diagnosis & \\
\hline MMT-m at interview & \\
\hline ALSFRS-r at interview & \\
\hline
\end{tabular}

\section{Comorbidities:}

\begin{tabular}{|l|l|}
\hline Heart dysfunction & \\
\hline Hypertension & \\
\hline Vascular dysfunction & \\
\hline Hematological dysfunction & \\
\hline Respiratory dysfunction (lung, bronchus, trachea, larynx) & \\
\hline EENT impairment (eye, ear, nose, throat) & \\
\hline $\begin{array}{l}\text { Gastroenteric dysfunction (esophagus, stomach, duodenum, gallbladder, pancreas, bowel, } \\
\text { hernias) }\end{array}$ & \\
\hline Liver dysfunction & \\
\hline Kidney dysfunction & \\
\hline Other genitourinary dysfunction (ureters, bladder, urethra, prostate, genitals) & \\
\hline Muscle-skeletal-skin impairment & \\
\hline Neurological dysfunction (brain, spinal cord, nerves) & \\
\hline Endocrine-Metabolic dysfunction (diabetes, thyroid) & \\
\hline Psychiatric/behavioral disorders (depression, anxiety, agitation/delirium, psychosis) & \\
\hline Infections & \\
\hline Autoimmune diseases & \\
\hline Tumors & \\
\hline Other & \\
\hline
\end{tabular}

\section{Allergological anamnesis:}

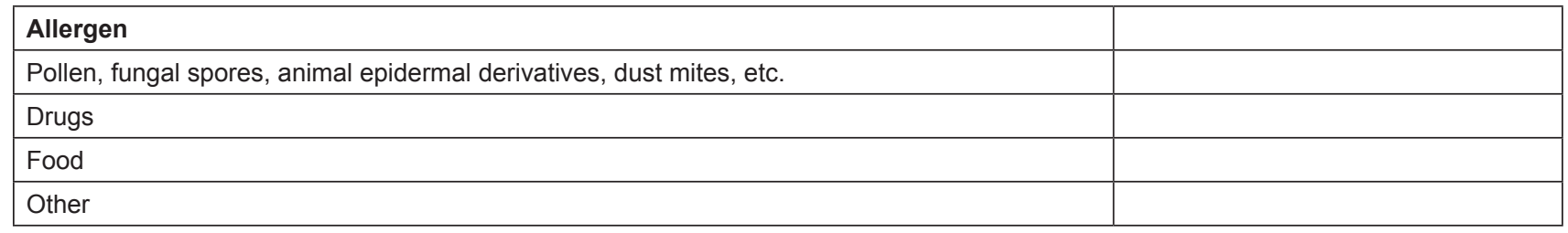

Type of allergic reaction:

Figure SI (Continued) 


\section{Current pharmacological anamnesis}

\begin{tabular}{|c|c|c|c|}
\hline Class of drug & $\square$ & Drug & Dosage \\
\hline \multicolumn{4}{|l|}{ 1) Corticosteroids } \\
\hline \multicolumn{4}{|l|}{ 2) NSAIDs } \\
\hline \multicolumn{4}{|c|}{ 3) Lipid-lowering agents } \\
\hline \multicolumn{4}{|l|}{ 4) Antidiabetics } \\
\hline \multicolumn{4}{|l|}{ 5) Antiarrhythmics } \\
\hline \multicolumn{4}{|l|}{ 6) Antihypertensive } \\
\hline \multicolumn{4}{|l|}{ 7) Benzodiazepines } \\
\hline \multicolumn{4}{|l|}{ 8) Antidepressive } \\
\hline \multicolumn{4}{|l|}{ 9) Antipsychotics } \\
\hline \multicolumn{4}{|l|}{ 10) Anticholinergic } \\
\hline \multicolumn{4}{|l|}{ 11) Antiparkinsonian } \\
\hline \multicolumn{4}{|c|}{ 12) Antiepileptic drugs } \\
\hline \multicolumn{4}{|l|}{ 13) Opioid analgesics } \\
\hline \multicolumn{4}{|c|}{ 14) Supplements/vitamins } \\
\hline \multicolumn{4}{|l|}{ 15) Bronchodilators } \\
\hline \multicolumn{4}{|l|}{ 16) Antihistamines } \\
\hline 17) Other & & & \\
\hline
\end{tabular}

Did you experience swallowing difficulties to assume therapies?|_|Yes |_|No

\section{Investigations on the use of riluzole:}

- What formulation of riluzole do you assume?

I__ Tablets |_ Oral suspension |_ None

Is riluzole tablet assumed from?

Diagnosis |_ Other date |_______

If "other date" indicate the reason

- Is riluzole oral suspension assumed from?

Diagnosis | _ O Other date |____ ___

If "other date" indicate the reason

How long?

- Tablets

- Oral suspension

- Did you always use the same formulation of riluzole? Yes |_ $\mid$ No |_

If no, what formulation of riluzole has been used before?

I__ Tablets |__ Oral suspension

Why did you change the formulation of riluzole?
I__ Dysphagia
I__ Preference for oral suspension
L_ Side effects
I_l Other
I__ Preference for tablets

Figure SI (Continued) 


\section{Investigations on the use of riluzole tablets:}

Have you been prescribed riluzole tablet $50 \mathrm{mg}$ as the first prescription of riluzole?

Yes |__ No |__

If no, why?

If you assume riluzole tablet $50 \mathrm{mg}$, have you received tablet prescription twice daily?

Yes |_ No I__

Do you taking the tablets twice daily? Yes |_ $\mid$ No |_ $\mid$

Are there signs/symptoms associated with the riluzole intake?

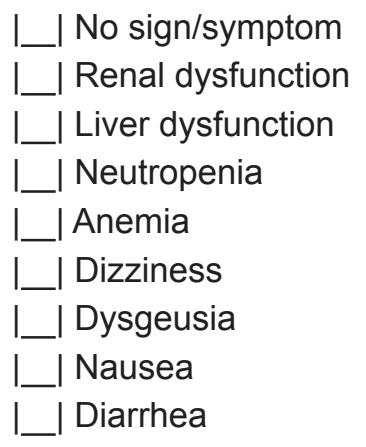

I__ Abdominal pain
I__ Oral paresthesia
I__ Headache
I_ Other

If yes, do you stop it? Yes |__ No I__

If no stop, do you take at same posology? Yes |_ I No |__

If you change the posology, indicate the $\underline{\mathrm{mg} / \mathrm{die}}$ are you currently taking

Have you ever missed taking tablets? I_ | Yes |_ | No

If yes why?

Do you experience swallowing difficulties when taking the tablet?

I_ Yes |_ No

If yes, did you talk to the healthcare staff? |_| Yes I__ No

Have you been offered a "oral suspension" therapeutic change?

I_ Yes |_ No

Do you think that riluzolo tablet might have altered your taste sensitivity?

I_|Yes |_ No |__ Maybe

\section{Investigations on the use of riluzole oral suspension}

Have you been prescribed with oral suspension from the first prescription of riluzole? Yes |_ $\mid$ No |_

Did you take riluzole tablets before? Yes |_ $\mid$ No |__

If yes, since when are you taking oral suspension? (date) |_____

Why the oral suspension has been prescribed?

I__ Dysphagia

I__ Swallowing fear

L__ Preference for oral suspension

I__ Preference for tablets

I_l Other

Figure SI (Continued) 
Do you take $10 \mathrm{~mL}$ oral suspension twice daily? Yes | _ No | _ _

Are there signs/symptoms associated with the riluzole intake?

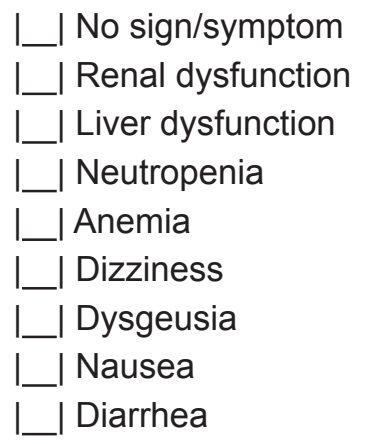

|__bdominal pain
|__ Oral paresthesia
|__ Headache
I_ Other

If yes, do you stop it? Yes |_ No |__

If no stop, do you take at same posology? Yes |_ $\mid$ No |_

If you change the posology, indicate the $\mathrm{mg} / \mathrm{die}$ are you currently taking |

How do you judge the consistency of oral suspension?

___ Pleasant |__ Unpleasant |__ Indifferent

How do you judge the fragrance of oral suspension?

I__ Pleasant |__ Unpleasant |__ Indifferent

How do you judge the color of oral suspension?

I__ Pleasant |__ Unpleasant |__ Indifferent

How do you judge the flavor of oral suspension?

I__ Pleasant |__ Unpleasant |__ Indifferent

Have you ever missed taking oral suspension?

If yes why?

Do you think that riluzole oral suspension might have altered your taste sensitivity? I_|Yes |__ No |_| Maybe

Figure SI Questionnaire on clinical and pharmacological features related to ALS and comorbidities.

Abbreviations: ALS, amyotrophic lateral sclerosis; ALSFRS-r, ALS Functional Rating Scale - revised; MMT-m, Manual Muscle Testing - medium; NSAIDs, nonsteroidal anti-inflammatory drugs.

\section{Publish your work in this journal}

Neuropsychiatric Disease and Treatment is an international, peerreviewed journal of clinical therapeutics and pharmacology focusing on concise rapid reporting of clinical or pre-clinical studies on a range of neuropsychiatric and neurological disorders. This journal is indexed on PubMed Central, the 'PsycINFO' database and CAS, and is the official journal of The International Neuropsychiatric Association (INA). The manuscript management system is completely online and includes a very quick and fair peer-review system, which is all easy to use. Visit http://www.dovepress.com/testimonials.php to read real quotes from published authors. 